Programa de Pós-Graduação em Engenharia de Produção - PPGEP

Laboratório de Qualidade de Vida - LaQVida

Universidade Tecnológica Federal do Paraná - UTFPR

Ponta Grossa - PR - Brasil

v. 04 , n. 01, jan./jun. 2012, p. 36-46

DOI: $10.3895 / \mathrm{S} 2175-08582012000100005$

\section{REVISTA BRASILEIRA DE QUALIDADE DE VIDA}

\title{
Qualidade de vida dos profissionais de enfermagem
}

\author{
Quality of life of the nursing professionals
}

\author{
Técia Maria Santos Carneiro e Cordeiro \\ Universidade Federal da Bahia - UFBA - Salvador - Brasil \\ teciamarya@yahoo.com.br
}

\begin{abstract}
Resumo
Considerando a qualidade de vida (QV) dos profissionais de enfermagem como fundamental para sua saúde e qualidade da assistência prestada, os objetivos deste estudo são: verificar a qualidade metodológica das produções científicas pesquisadas; descrever o perfil das produções científicas acerca da QV dos profissionais de enfermagem; e identificar nas produções científicas os fatores interferentes na QV dos profissionais de enfermagem e os aspectos determinantes para QV. Tratase de um estudo bibliográfico com abordagem qualitativa de cunho descritivo exploratório. A busca de dados deu-se nas bases eletrônicas da BVS e SciELO. Seguiu os critérios de inclusão: a) artigos publicados na íntegra em português no período de janeiro de 1999 a maio de 2012; b) com profissionais de enfermagem como sujeitos dos estudos; c) temática de forma clara no título. Constituindo o corpus documental deste estudo quinze artigos científicos. Os dados foram analisados por meio da análise de conteúdo temática. Os resultados apontam que a qualidade metodológica dos estudos analisados apresenta parâmetros aceitáveis e o perfil da amostra confirma estudos já realizados. Os fatores interferentes na QV consistem em baixos salários, riscos ocupacionais, condições laborais precárias, (des)valorização profissional, (in)satisfação pelo trabalho. Os aspectos determinantes para QV referem-se à melhoria dos ambientes de trabalho, implementação de políticas públicas, programas de atenção a Saúde do Trabalhador, valorização profissional. Considera-se que para o cuidado ser prestado com qualidade, os profissionais de enfermagem necessitam de cuidados e valorização, o que contribui para a satisfação profissional e uma possível qualidade de vida.
\end{abstract}

Palavras-chave: qualidade de vida, profissional de enfermagem, satisfação profissional, condições de trabalho.

\footnotetext{
Abstract

Considering the quality of life (QL) of the nursing professionals as fundamental to their health and quality of assistance provided, the objectives of this study are: to assess the methodological quality of scientific production surveyed; to describe the profile of scientific production about the QL of professionals nursing; and identify in the scientific production the interfering factors on QL of nursing professionals and the determining aspects for QL. This is a bibliographical study with a qualitative approach to a nature descriptive exploratory. The search for data was the electronic databases of the VHL and SciELO. Was followed the inclusion criteria: a) articles published in full in Portuguese in the period January 1999 to May 2012; b) with nursing professionals as subjects of study; c) thematic to clearly in the title. Constituting the documents corpus of this study fifteen scientific papers. The data were analyzed using a thematic content analysis. The results indicate that the methodological quality of studies presented analyzed acceptable parameters and the profile
} 
of the sample confirms existing studies. The interfering factors in QL consists of low salaries, occupational hazards, poor working conditions, professional (de)valuation, (dis)satisfaction with the work. The determining factors for QL refers to the improvement of work environments, implementation of public policies, programs Health Care Worker's, professional valuation. Is considered that to be provided with quality care, nursing professionals need care and recovery, which contributes to job satisfaction and a life quality possible.

Keywords: quality of life, nursing professionals, job satisfaction, working conditions.

\section{Introdução}

A Qualidade de Vida (QV) tem sido estudada em vários campos, principalmente em estudos associados às condições de trabalho no campo da saúde (FOGAÇA; CARVALHO; MARTINS, 2010). Temas relacionados ao trabalho e QV dos profissionais de enfermagem vêm sendo abordados em diferentes áreas, perspectivas e métodos (KIMURA; CARANDINA, 2009).

O grupo de QV da Organização Mundial de Saúde (OMS) define QV como "a percepção do indivíduo de sua posição na vida, no contexto da cultura e sistema de valores nos quais ele vive e em relação aos seus objetivos, expectativas, padrões e preocupações" (THE WHOQOL GROUP, 1995). Esta definição é subjetiva por envolver aspectos culturais, religiosos, pessoais, sociais, ambientais, familiares e do trabalho.

Para mensurar a QV são usados muitos instrumentos através dos indicadores que permeiam amplamente a vida de um ser humano (ALVES, 2011). Os principais instrumentos usados em pesquisas com profissionais de enfermagem são: World Health Organization Quality of Life (WHOQOL) -100 / Bref, Escala de Qualidade de Vida de Flanagan, Formulário Abreviado da Avaliação de Saúde 36 (SF-36) e o Índice de Satisfação Profissional (ISF).

A satisfação profissional está sendo abordada no Brasil e no exterior por ser considerada um dos indicadores de Qualidade de Vida no Trabalho (QVT) (SCHMIDT; DANTAS, 2006b). Segundo Chiavenato (2008), a QVT é o conjunto de características do trabalhador e aspectos no contexto do trabalho como a atuação sistêmica das características organizacionais e individuais que configuram o mundo do trabalho.

De acordo com Lentz et al. (2000), vários autores têm conceituado QV como um indicador global de bem-estar e satisfação de vida dos profissionais de enfermagem. A valorização, o desenvolvimento e o reconhecimento são fundamentais para a realização pessoal, assim como, a realização depende também da valorização, do desenvolvimento e do reconhecimento que é oferecido através de uma relação humanística influenciando na QV dos profissionais da enfermagem. Visto que as atividades de enfermagem assistenciais e de docência devem ser desenvolvidas com prazer, e não apenas por obrigação, para promover a satisfação pessoal, de vida, profissional e determinar a QV. A Figura 1 representa esta idéia configurada por Lentz et al. (2000):

Figura 1 - Representação dos fatores que influenciam na qualidade de vida dos profissionais de enfermagem

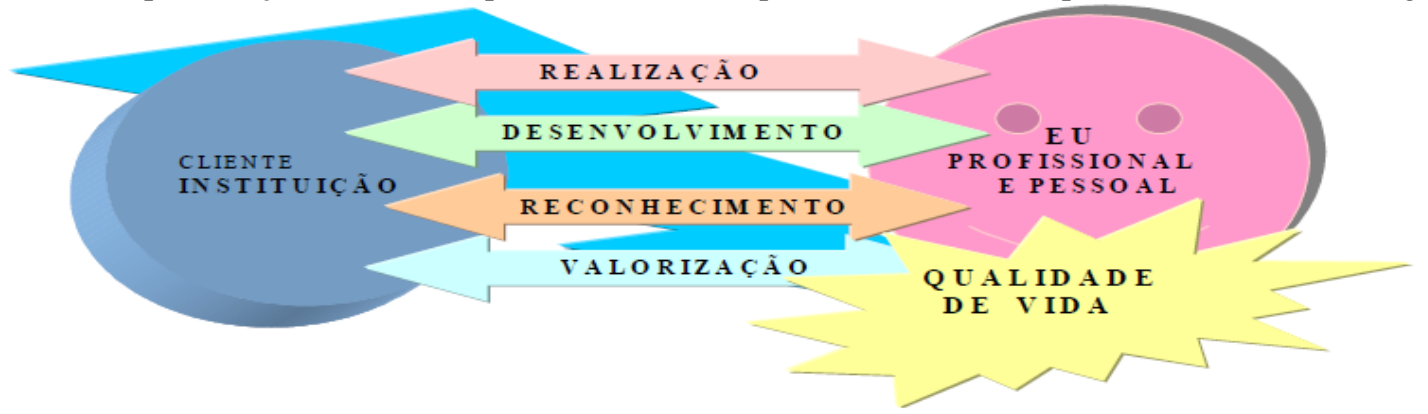

Fonte: Lentz et al. (2000). 
Alguns pesquisadores como Kimura e Carandina (2009), Fogaça, Carvalho e Martins (2010) e Fernandes et al. (2012) têm desenvolvido estudos para mensurar a QV dos profissionais de enfermagem e investigar os fatores interferentes a fim de propor medidas estratégicas para melhoria da QV desses profissionais, observado que esses fatores não dependem apenas dos profissionais e sim de todo o contexto que envolve a sua vida pessoal e profissional.

Diante do exposto, considerando a QV dos profissionais de enfermagem como fundamental para sua saúde e qualidade da assistência prestada, os objetivos do presente estudo são: verificar a qualidade metodológica das produções científicas pesquisadas; descrever o perfil das produções científicas acerca da QV dos profissionais de enfermagem e, identificar nas produções científicas os fatores interferentes na QV dos profissionais de enfermagem e os aspectos determinantes para QV.

\section{Metodologia}

Trata-se de um estudo bibliográfico com abordagem qualitativa de cunho descritivo exploratório. A busca de dados deu-se nas bases eletrônicas da Biblioteca Virtual em Saúde (BVS) e Scientific Eletronic Library Online (SciELO Brasil) com as palavras-chave nas respectivas bases: BVS - 'qualidade de vida dos profissionais de enfermagem' e SciELO - 'qualidade de vida', 'enfermagem' e 'docente'. Na base do SciELO recorreu-se ao operador lógico and para combinação das palavras no rastreamento das produções científicas.

Foram definidos os seguintes critérios de inclusão: a) artigos publicados na íntegra em português no período de janeiro de 1999 a maio de 2012; b) com profissionais de enfermagem (auxiliares de enfermagem, técnicos e enfermeiros) como sujeitos dos estudos; c) temática de forma clara no título. Excluíram-se os artigos repetidos e os que tinham disponível nas bases eletrônicas apenas os resumos, perfazendo o corpus documental deste estudo quinze artigos científicos.

Para avaliar a qualidade metodológica dos artigos que compõe a amostra foi utilizado os Critérios de Verificação da Qualidade Metodológica adaptado por Mascarenhas e Fernandes (2011), considerando qualidade + (mais) o artigo que contemplasse na íntegra a descrição do item de verificação, +/- (mais ou menos) para o atendimento parcial, - (menos) o não atendimento e NA (não se aplica) aos estudos que não apresentavam compatibilidade com o item verificado. $\mathrm{O}$ Quadro 1 apresenta os critérios avaliados:

\begin{tabular}{|c|c|c|}
\hline Item & Critério & Descrição \\
\hline A & Objetivo & $\begin{array}{l}\text { Definição clara e concisa do que se buscou avaliar com o } \\
\text { estudo. }\end{array}$ \\
\hline B & Seleção da amostra & $\begin{array}{l}\text { Adoção de critérios de aleatoriedade para seleção dos } \\
\text { sujeitos/grupos de sujeitos para o estudo. }\end{array}$ \\
\hline $\mathrm{C}$ & Inclusão/Exclusão & $\begin{array}{l}\text { Clareza e objetividade nos critérios definidos para seleção } \\
\text { dos sujeitos. }\end{array}$ \\
\hline $\mathrm{D}$ & Participação dos sujeitos & $\begin{array}{l}\text { Frequência de pelo menos } 80 \% \text { dos sujeitos para estudos até } \\
3 \text { meses de acompanhamento e de pelo menos } 60 \% \text { para } \\
\text { estudos acima de } 3 \text { meses para conclusão. }\end{array}$ \\
\hline $\mathrm{E}$ & Instrumentos de coleta de dados & $\begin{array}{l}\text { Uso de instrumento validado ou referência a validação do } \\
\text { instrumento aplicado. }\end{array}$ \\
\hline $\mathrm{F}$ & Coleta dos dados & $\begin{array}{l}\text { Referência a impessoalidade ou treinamento dos } \\
\text { pesquisadores para aplicação de questionário ou realização } \\
\text { de medidas diretas. }\end{array}$ \\
\hline G & Tratamento estatístico & $\begin{array}{l}\text { Utilização de técnicas estatísticas compatíveis com modelo de } \\
\text { estudo de pesquisa. }\end{array}$ \\
\hline $\mathrm{H}$ & Pontos fortes e fracos & Referências a possíveis vantagens e desvantagens do estudo. \\
\hline । & Conclusão & $\begin{array}{l}\text { Clareza e objetividade em relação aos achados e objetivos do } \\
\text { estudo. }\end{array}$ \\
\hline
\end{tabular}

Fonte: Mascarenhas e Fernandes (2011). 
A análise dos dados foi feita segundo a análise de conteúdo descrita por Bardin (1979), que diz respeito a um conjunto de técnicas de análise que visa obter por procedimentos sistemáticos e objetivos o conteúdo das mensagens e indicadores que permitam a inferência de conhecimentos. Em especial, utilizou-se a análise temática que segundo Minayo (2010) comporta um feixe de relações e podem ser graficamente apresentada através de palavras, frases ou resumos. Seguiramse três etapas: pré-análise, exploração do material e tratamento dos resultados obtidos e interpretação.

Dessa forma, os dados foram apresentados em relação ao perfil do corpus documental em estudo, à qualidade metodológica das produções científicas, os fatores interferentes na QV e aspectos determinantes da QV dos profissionais de enfermagem.

\section{Resultados e discussões}

\subsection{Perfil das produções científicas (corpus documental)}

O Quadro 2 apresenta o perfil das produções científicas que compõe o corpus documental em estudo.

Quadro 2 - Corpus documental

\begin{tabular}{|c|c|c|c|c|c|c|c|c|c|c|c|c|}
\hline $\begin{array}{c}\mathrm{N}^{\mathbf{o}} \\
\text { Artigo }\end{array}$ & Ano & Estado & Revista & EA & ED & $\begin{array}{c}\text { Categoria } \\
\text { Prof. }\end{array}$ & Sexo & $\begin{array}{l}\text { Idade } \\
\text { (anos) }\end{array}$ & $\mathrm{C} / \mathrm{S}$ & $\begin{array}{c}\text { TS } \\
(\operatorname{anos})\end{array}$ & $\mathbf{S M}$ & $\begin{array}{l}+ \text { de } \\
1 \mathrm{VE}\end{array}$ \\
\hline 1 & 2004 & $\mathrm{RJ}$ & $\begin{array}{c}\text { Esc. } \\
\text { Anna } \\
\text { Nery R } \\
\text { Enferm. }\end{array}$ & $X$ & - & $\begin{array}{c}7 \text { Enf. } \\
3 \mathrm{TE} \\
24 \mathrm{AE}\end{array}$ & $>$ Fem. & $35-55$ & - & $10-19$ & $1-3$ & $\mathrm{X}$ \\
\hline 2 & 1999 & $\mathrm{RS}$ & $\begin{array}{c}\text { Rev. } \\
\text { Esc. } \\
\text { Enferm. } \\
\text { USP }\end{array}$ & - & $X$ & 4 Enf. & $>$ Fem. & - & $>\mathrm{C}$ & $>3$ & - & - \\
\hline 3 & 2010 & MG & $\begin{array}{c}\text { Texto e } \\
\text { Contexto } \\
\text { Enferm. }\end{array}$ & $\mathrm{X}$ & - & 90 Enf. & $>$ Fem. & $20-30$ & $>\mathrm{S}$ & $<10$ & - & - \\
\hline 4 & 2009 & PB & $\begin{array}{c}\text { Rev. } \\
\text { Eletr. } \\
\text { Enf. }\end{array}$ & $X$ & - & 12 Enf. & $>$ Fem. & $25-55$ & $>\mathrm{C}$ & $1-5$ & $>10$ & $X$ \\
\hline 5 & 2000 & SC/RS & $\begin{array}{c}\text { Rev. } \\
\text { Latino- } \\
\text { am. } \\
\text { Enferm. }\end{array}$ & $\mathrm{X}$ & - & $\begin{array}{c}14 \text { Enf. } \\
7 \mathrm{TE} \\
31 \mathrm{AE}\end{array}$ & $>$ Fem. & $30-35$ & $>\mathrm{C}$ & $2-6$ & - & - \\
\hline 6 & 2010 & SP & $\begin{array}{l}\text { Rev. } \\
\text { Esc. } \\
\text { Enferm. } \\
\text { USP }\end{array}$ & $X$ & - & 20 Enf. & $>$ Fem. & $30-36$ & - & $4-6$ & - & - \\
\hline 7 & 2006 & PR & $\begin{array}{c}\text { Rev. } \\
\text { Latino- } \\
\text { am. } \\
\text { Enferm. }\end{array}$ & $\mathrm{X}$ & - & $\begin{array}{c}12 \mathrm{Enf} . \\
1 \mathrm{TE} \\
73 \mathrm{AE} \\
18 \mathrm{ATE}\end{array}$ & $>$ Fem. & $21-60$ & $>\mathrm{C}$ & - & - & - \\
\hline 8 & 2007 & SP & $\begin{array}{c}\text { Acta } \\
\text { Paul. } \\
\text { Enferm. }\end{array}$ & $\mathrm{X}$ & - & $\begin{array}{c}126 \mathrm{AE} / \\
\mathrm{TE}\end{array}$ & $>$ Fem. & $20-56$ & $>S$ & - & $2-6$ & $\mathrm{X}$ \\
\hline 9 & 2007 & RJ & $\begin{array}{c}\text { Esc. } \\
\text { Anna } \\
\text { Nery R } \\
\text { Enferm. }\end{array}$ & $\mathrm{X}$ & - & $\begin{array}{c}7 \text { Enf. } \\
3 \mathrm{TE} \\
24 \mathrm{AE}\end{array}$ & $>$ Fem. & $35-55$ & - & $10-19$ & $1-3$ & $\mathrm{X}$ \\
\hline 10 & 2009 & SP & $\begin{array}{l}\text { Rev. } \\
\text { Esc. } \\
\text { Enferm. } \\
\text { USP }\end{array}$ & $X$ & - & 348 Enf. & $>$ Fem. & $21-64$ & $>\mathrm{S}$ & $5-7$ & $3-4$ & - \\
\hline
\end{tabular}




\begin{tabular}{|c|c|c|c|c|c|c|c|c|c|c|c|c|}
\hline $\begin{array}{c}\mathbf{N}^{\mathbf{o}} \\
\text { Artigo }\end{array}$ & Ano & Estado & Revista & EA & ED & $\begin{array}{c}\text { Categoria } \\
\text { Prof. }\end{array}$ & Sexo & $\begin{array}{l}\text { Idade } \\
\text { (anos) }\end{array}$ & $\mathrm{C} / \mathrm{S}$ & $\begin{array}{c}\text { TS } \\
\text { (anos) }\end{array}$ & SM & $\begin{array}{l}+ \text { de } \\
1 \mathrm{VE}\end{array}$ \\
\hline 11 & 2004 & SP & $\begin{array}{c}\text { Rev. } \\
\text { Latino- } \\
\text { am. } \\
\text { Enferm. }\end{array}$ & - & $\mathrm{X}$ & 15 Enf. & $>$ Fem. & $33-61$ & - & 6-18 & - & $\mathrm{X}$ \\
\hline 12 & 2006 & SP & $\begin{array}{c}\text { Rev. } \\
\text { Ciênc. } \\
\text { Méd. }\end{array}$ & $\mathrm{X}$ & - & $\begin{array}{l}4 \text { Enf. } \\
42 \mathrm{AE}\end{array}$ & $>$ Fem. & $20-40$ & $>\mathrm{C}$ & $2-11$ & - & - \\
\hline 13 & 2006 & PR & $\begin{array}{l}\text { Rev. } \\
\text { Gaúcha } \\
\text { Enferm. }\end{array}$ & $\mathrm{X}$ & - & $\begin{array}{c}12 \mathrm{Enf} . \\
1 \mathrm{TE} \\
73 \mathrm{AE} \\
17 \mathrm{ATE}\end{array}$ & $>\mathrm{Fem}$. & $21-60$ & $>\mathrm{C}$ & $2-15$ & $1-3$ & - \\
\hline 14 & 2012 & SP & $\begin{array}{c}\text { Acta } \\
\text { Paul. } \\
\text { Enferm. }\end{array}$ & $\mathrm{X}$ & - & 8 Enf. & $>$ Fem. & $25-49$ & - & $1-21$ & - & $\mathrm{X}$ \\
\hline 15 & 2012 & MG & $\begin{array}{c}\text { Rev. } \\
\text { Esc. } \\
\text { Enferm. } \\
\text { USP }\end{array}$ & $\mathrm{X}$ & - & 90 Enf. & $>$ Fem. & $20-30$ & - & - & 4-8 & $\mathrm{X}$ \\
\hline
\end{tabular}

Legenda: EA: Enfermeiro Assistencialista; ED: Enfermeiro Docente; Prof.: Profissional; C/S: Casado/Solteiro; TS: Tempo de Serviço; SM: Salário Mínimo; + de 1 VE: Mais de um Vínculo Empregatício; Enf.: Enfermeiro; TE: Técnico de Enfermagem; AE: Auxiliar de Enfermagem; ATE: Atendente de Enfermagem.

Fonte: Autoria própria (2012).

A maioria dos estudos $(n=11)$ foi publicada no período de 2006-2012, realizadas no estado de São Paulo $(n=6)$, com abordagem nos enfermeiros assistencialistas $(n=13)$ e publicados em periódicos de enfermagem ( $\mathrm{n}=14)$, em específico nas Revistas da Escola de Enfermagem da USP $(\mathrm{n}=4)$ e na Revista Latino-americana de Enfermagem $(\mathrm{n}=3)$.

Os participantes dos estudos estão caracterizados em sua maioria: do sexo feminino $(n=15)$, com idade entre 20-50 anos, casados $(n=6)$, auxiliares de enfermagem $(n=7)$, em exercício profissional menor que 15 anos $(n=9)$, com remuneração de 1-4 salários mínimos e a metade com mais de um vínculo empregatício $(n=7)$.

Dessa maneira, o interesse à pesquisa sobre QV dos profissionais de enfermagem se manteve estável nos últimos anos. Sendo, que se fazem necessários maiores investimentos em pesquisas, pelas condições precárias de trabalho que são impostos a esses profissionais, em relação aos vínculos precários, recursos escassos e relações interpessoais inadequadas, como afirmam Farias e Zeitoune (2004) e Fernandes et al. (2012).

Esta temática precisa ser desenvolvida com maior expansão nas regiões norte, centro-oeste e nordeste, pela inexistência e baixo número de produções respectivamente, além de um enfoque maior com os enfermeiros docentes que se submetem as mesmas condições de trabalho dos assistencialistas.

As características dos profissionais de enfermagem nas produções científicas confirmam os achados em outras pesquisas. Referente ao sexo predomina o feminino, o que reporta uma característica histórica ligada à profissão da enfermagem: o cuidado. $\mathrm{O}$ ato de cuidar sempre associado à figura feminina (FOGAÇA; CARVALHO; MARTINS, 2010; DAUBERMANN; TONETE, 2012; FERNANDES et al., 2012).

Em relação à faixa etária, várias pesquisas apontam idades entre 20-40 anos formando um grupo de adultos jovens (TALHAFERRO; BARBOZA; DOMINGOS, 2006). Mas, os dados do presente estudo revela uma parcela dos profissionais acima dos 40 anos e próximo aos 60 anos $(\mathrm{n}=4)$, o que pode se relacionar a QV comprometida pelas condições laborais impostas e a idade avançada. (COSTA; MORITA; MARTINEZ, 2000; REZENDE, 2003; SCHMIDT; DANTAS, 2006a).

A categoria profissional que se destaca em maior número é a de auxiliares de enfermagem, o que chama atenção que essa formação encontra-se extinta e os profissionais que atuam como auxiliares só podem renovar sua inscrição no conselho duas vezes, porque na terceira faz 
necessário complementar o curso para técnico de enfermagem, a fim de incentivar os profissionais a continuar sua formação (COFEN, 2007). Observa-se em dois estudos realizados no estado do Paraná um quantitativo de atendentes de enfermagem, profissão essa com contratação extinta desde 1994, com a Lei Federal ${ }^{\circ}$ 8.967. Segundo Fakih et al. (1999) isso decorreu pela carência de recursos humanos de nível médio para a enfermagem e os baixos salários.

De acordo com Fernandes et al. (2010), os profissionais com menos de seis anos de tempo de serviço estão atuando nas Estratégias de Saúde da Família (ESF) e com mais de sete anos nos hospitais e na docência. Isso pode está associado ao maior conhecimento com menor tempo de formação acerca das bases e princípios da ESF, que obteve expansão recente e inserção nos currículos, além da exigência de carga horária extensa e salários baixos.

A remuneração baixa referida nas produções científicas está de acordo à multiplicidade de vínculos empregatícios, contribuindo para a assistência desqualificada e interferência na QV dos profissionais de enfermagem.

\subsection{Qualidade metodológica das produções científicas}

As produções científicas selecionadas foram quinze, as quais foram avaliadas segundo os Critérios de Verificação da Qualidade Metodológica adaptado por Mascarenhas e Fernandes (2011). A Tabela 1 apresenta a verificação segundo cada item avaliado:

Tabela 1 - Análise da qualidade metodológica das produções científicas em estudo

\begin{tabular}{|c|c|c|c|c|c|c|c|c|c|c|c|}
\hline \multirow{2}{*}{$\begin{array}{c}\mathrm{N}^{\mathbf{0}} \\
\text { Artigo }\end{array}$} & \multirow[t]{2}{*}{ Autor / data } & \multicolumn{9}{|c|}{ Critérios de qualidade metodológica } & \multirow[t]{2}{*}{ Total } \\
\hline & & $\mathbf{A}$ & $\mathbf{B}$ & $\mathbf{C}$ & D & $\mathbf{E}$ & $\mathbf{F}$ & $\mathbf{G}$ & $\mathbf{H}$ & I & \\
\hline 1 & Farias; Zeitoune / 2004 & + & NA & + & + & $+/-$ & + & NA & - & + & 5 \\
\hline 2 & $\begin{array}{c}\text { Beck; Budó; Gonzales / } \\
1999\end{array}$ & $+/-$ & + & $+/-$ & + & + & + & NA & - & + & 5 \\
\hline 3 & Fernandes et al. / 2010 & + & + & + & + & + & + & + & $+/-$ & $+/-$ & 7 \\
\hline 4 & $\begin{array}{l}\text { Araújo; Soares; Henriques / } \\
2009\end{array}$ & + & - & - & + & + & + & NA & $+/-$ & + & 5 \\
\hline 5 & Lentz et al. / 2000 & + & + & + & + & + & + & $+/-$ & - & + & 7 \\
\hline 6 & $\begin{array}{l}\text { Fogaça; Carvalho; Martins / } \\
\qquad 2010\end{array}$ & + & + & + & - & + & $+/-$ & + & + & $+/-$ & 6 \\
\hline 7 & Schmidt; Dantas / 2006 & + & - & $+/-$ & + & + & + & + & $+/-$ & $+/-$ & 5 \\
\hline 8 & $\begin{array}{l}\text { Paschoa; Zanei; Whitaker / } \\
2007\end{array}$ & + & NA & - & + & + & + & + & - & + & 6 \\
\hline 9 & Farias; Zeitoune / 2007 & + & + & + & + & $+/-$ & + & NA & - & + & 6 \\
\hline 10 & Kimura; Carandina / 2009 & + & + & + & + & $+/-$ & + & + & + & + & 8 \\
\hline 11 & Rocha; Feli / 2004 & + & + & + & + & $+/-$ & + & NA & - & + & 6 \\
\hline 12 & $\begin{array}{l}\text { Talhaferro; Barboza; } \\
\text { Domingos / } 2006\end{array}$ & + & + & + & + & + & + & + & - & + & 8 \\
\hline 13 & Schmidt; Dantas / 2006 & + & $+/-$ & - & + & + & + & + & - & + & 6 \\
\hline 14 & Daubermann; Tonete / 2012 & + & + & + & + & $+/-$ & + & NA & - & + & 6 \\
\hline 15 & Fernandes et al. / 2012 & + & + & - & + & + & + & + & - & + & 7 \\
\hline
\end{tabular}

Legenda: + (atende na íntegra a descrição); +/- (atende parcialmente); - (não atende); NA (não de aplica).

Fonte: Autoria própria (2012).

Comparando com o estudo realizado por Mascarenhas e Fernandes (2011) sobre a associação de atividades físicas de lazer e distúrbios muscoesqueléticos relacionados ao trabalho (tamanho da amostra: quinze), que obteve: uma produção científica atendendo a todos os parâmetros com maior escore $(n=9)$; três com menor escore $(n=4)$; e doze com boa taxa de participação dos sujeitos $(>80 \%)$. Observa-se uma diferença ao presente estudo, que nenhuma produção científica atendeu a todos os critérios de qualidade metodológica avaliados, duas produções atenderam ao maior escore $(n=8)$ e quatro atenderam ao menor escore $(n=5)$. Apenas um estudo apresentou menos de $80 \%$ da participação dos sujeitos (50\%).

Isto se pode relacionar a dissociação das temáticas, apesar de estarem relacionadas à Saúde do Trabalhador. Contanto, necessita-se apenas, que os pesquisadores sejam mais claros, concisos e 
coerentes em cada etapa de suas pesquisas e conciliando sempre a idéia da temática com os objetivos, os resultados e as conclusões.

\subsection{Fatores interferentes na $Q V$ e aspectos determinantes para $Q V$ dos profissionais de enfermagem}

A QV é determinada por parâmetros sócio-ambientais como transporte, segurança, assistência médica, condições de trabalho, educação, meio ambiente e estilo de vida (GORDIA et al., 2011). Baseada nesta afirmação pode-se dizer que o trabalho e a saúde é um mediador da QV dos profissionais de enfermagem e através deles que se podem lançar estratégias para promover a QV.

Os Quadros 3 e 4, respectivamente, apresentam os fatores que interferem na QV dos profissionais de enfermagem (PE) e os aspectos necessários para determinar a QV desses profissionais, baseados nos resultados das produções científicas estudadas.

Quadro 3 - Fatores interferentes na QV dos PE

\begin{tabular}{|c|c|}
\hline \multicolumn{2}{|c|}{ Fatores interferentes } \\
\hline Baixos salários & Acúmulo de atividades \\
\hline Riscos ocupacionais & Pouco tempo para o lazer \\
\hline Multiplicidade de empregos & Interferência laboral na vida familiar \\
\hline Condições laborais precárias & Sofrimento psíquico \\
\hline Falta de segurança & (In) Satisfação pelo trabalho \\
\hline Comunicações e atualizações insuficientes & Regime de trabalho \\
\hline (Des) Valorização profissional & Normas Organizacionais \\
\hline Abandono do autocuidado & Falta de privacidade \\
\hline
\end{tabular}

Fonte: Conteúdo retirado das produções científicas selecionadas neste estudo (2012).

\begin{tabular}{|c|}
\multicolumn{1}{|c|}{ Quadro 4 - Aspectos determinantes para a QV dos PE } \\
\hline Aspectos determinantes \\
\hline Melhoria, reestruturação e modernização dos ambientes de trabalho \\
\hline Melhoria na formação profissional \\
\hline Políticas públicas \\
\hline Projeto político pedagógico \\
\hline Programas de atenção à Saúde do Trabalhador \\
\hline Programas de segurança \\
\hline Salários condizentes \\
\hline Valorização profissional \\
\hline Valorização do ser e fazer da enfermagem \\
\hline
\end{tabular}

Fonte: Conteúdo retirado das produções científicas selecionadas neste estudo (2012).

Como apresentado no Quadro 3, os baixos salários interferem na QV dos PE por não serem condizentes com as categoriais profissionais da enfermagem, contribuindo para a multiplicidade de empregos e expondo o profissional a riscos ocupacionais, desgaste físico e mental (FARIAS; ZEITOUNE, 2007; ARAÚJO; SOARES; HENRIQUES, 2009; DAUBERMANN; TONETE, 2012; FERNANDES et al., 2012).

Os riscos ocupacionais a que estão expostos os PE são associados aos recursos escassos e condições laborais precárias, como ambiente desestruturado, falta de segurança nas unidades de saúde, diversidade de papéis, que expõe os profissionais à problemas ergonômicos, risco de vida, comprometimento da saúde em geral (FARIAS; ZEITOUNE, 2004; ROCHA; FELLI, 2004; SCHMIDT; DANTAS, 2006b; FARIAS; ZEITOUNE, 2007; ARAÚJO; SOARES; HENRIQUES, 2009; KIMURA; CARANDINA, 2009; FERNANDES et al., 2012).

Observa-se que as comunicações e atualizações insuficientes também interferem na QV pela exposição às práticas e rotinas ultrapassadas que compromete a saúde profissional e a 
assistência prestada. Diante das precariedades propostas a esses profissionais, os mesmos não são valorizados dentro das instituições de trabalho, sendo que a enfermagem é fundamental para equipe de saúde (BECK; BUDÓ; GONZALES, 1999; LENTZ et al., 2000; FARIAS; ZEITOUNE, 2004; SCHMIDT; DANTAS, 2006b; DAUBERMANN; TONETE, 2012).

Desse modo, os profissionais de enfermagem prestam assistência ao outro e esquecem de si, abandona o autocuidado colaborando para o comprometimento da $\mathrm{QV}$, além do acúmulo de atividades e papéis na sociedade. A maioria desses profissionais é do sexo feminino e exercem a função de trabalhador, mãe, dona de casa, esposa, entre outras, sobrando pouco tempo para as atividades recreacionais, esportivas e para o lazer. Isso provoca uma situação desgastante pelas atividades laborais interferirem na vida familiar (ROCHA; FELLI, 2004; SCHMIDT; DANTAS, 2006a; ARAÚJO; SOARES; HENRIQUES, 2009; FERNANDES et al., 2010; DAUBERMANN; TONETE, 2012).

Com esses fatores, acompanhados do ritmo acelerado de produção, pressão no ambiente laboral, inexistência ou escassas pausas para descanso no trabalho, fragmentação de tarefas e desqualificação do trabalho realizado, os profissionais podem desenvolver um sofrimento psíquico que interfere na QV e provoca uma insatisfação profissional (TALHAFERRO; BARBOZA; DOMINGOS, 2006; SCHMIDT; DANTAS, 2006a; FOGAÇA; CARVALHO; MARTINS, 2010; FERNANDES et al., 2012).

Entretanto, a (in)satisfação profissional constitui um fator determinante na QV tanto no trabalho, quanto geral, mas, a maioria das produções científicas aponta a insatisfação pelos profissionais acerca do regime de trabalho, das normas organizacionais, da falta de privacidade no ambiente laboral e de todas as condições precárias que lhes são impostas para atuar nas funções de assistencialista e docência (BECK; BUDÓ; GONZALES, 1999; SCHMIDT; DANTAS, 2006b; KIMURA; CARANDINA, 2009; FERNANDES et al., 2012).

Diante desses resultados, são inúmeros os fatores que interferem na QV dos profissionais de enfermagem, entretanto, para que essa situação seja modificada se faz necessário maiores investimentos para melhoria, reestruturação e modernizações dos ambientes de trabalho e na formação profissional. Além de medidas, para implementar políticas públicas que contemplem valores, interesses básicos, subsídios para melhores condições de trabalho e um projeto político pedagógico coletivo que considere a carreira profissional e o desgaste desses trabalhadores (BECK; BUDÓ; GONZALES, 1999; FARIAS; ZEITOUNE, 2004; ROCHA; FELLI, 2004; SCHMIDT; DANTAS, 2006b; DAUBERMANN; TONETE, 2012; FERNANDES et al., 2012).

Também se fazem necessários programas voltados à atenção a Saúde do Trabalhador nos ambientes de trabalhos e programas de segurança dentro das instituições considerando a estabilidade, formação, ambiente, assistência prestada, o bem-estar e a QVT dos profissionais de enfermagem. Os salários devem ser condizentes às atividades laborais desenvolvidas e à carga horária, enfim, essa profissão deve ser valorizada por sua função insubstituível nos serviços de saúde (BECK; BUDÓ; GONZALES, 1999; FARIAS; ZEITOUNE, 2004; SCHMIDT; DANTAS, 2006b; PASCHOA; ZANEI; WHITAKER, 2007; FARIAS; ZEITOUNE, 2007; FERNANDES et al., 2010; DAUBERMANN; TONETE, 2012).

Contudo, para que esses aspectos determinantes prevaleçam, os profissionais de enfermagem devem valorizar o ser e o fazer da enfermagem proporcionando uma QV satisfatória nas duas faces: o ambiente assistencial que abrange a promoção e recuperação da saúde e a docência, academia, na participação relevante na formação de profissionais da equipe de saúde (LENTZ et al., 2000; TALHAFERRO; BARBOZA; DOMINGOS, 2006; ARAÚJO; SOARES; HENRIQUES, 2009).

\section{Considerações Finais}

Diante dos resultados do presente estudo, pode-se afirmar que a QV é mediada pela saúde e pelo trabalho envolvendo todo o contexto de vida de um ser humano, como os aspectos culturais, sociais, religiosos, profissionais, pessoais, emocionais, psicológicos e ambientais. 
De acordo as produções científicas estudadas, a qualidade metodológica dos estudos sobre QV dos profissionais de enfermagem apresenta parâmetros aceitáveis e o perfil do corpus documental confirma resultados de estudos já realizados. Os fatores interferentes na QV dos profissionais de enfermagem consistem nos baixos salários, riscos ocupacionais, multiplicidade de empregos, condições laborais precárias, falta de segurança, (des)valorização profissional, comunicações e atualizações insuficientes, abandono do autocuidado, acúmulo de atividades, pouco tempo para o lazer, interferência laboral na vida familiar, sofrimento psíquico, (in)satisfação pelo trabalho, regime de trabalho, normas organizacionais e falta de privacidade.

Para que esses fatores sejam modificados os aspectos necessários para determinar a QV dos profissionais de enfermagem referem à melhoria, reestruturação e modernização dos ambientes de trabalho, melhoria na formação profissional, políticas públicas, projeto político pedagógico, programas de atenção à Saúde do Trabalhador, programas de segurança, salários condizentes, valorização profissional e do ser e fazer da enfermagem.

Considera-se relevante discutir essa temática sobre QV que envolve os profissionais de enfermagem, por esses trabalhadores está vivenciando propostas de cargos e funções que inclui salário, regime de trabalho e carga horária incompatível com a formação e manutenção da QV. A enfermagem é uma profissão relacionada à arte do cuidar, de fundamental importância nas instituições de saúde e na formação de profissionais para a equipe de saúde. Porém, para que o cuidado seja prestado com qualidade, os profissionais de enfermagem necessitam de cuidados e valorização, o que contribui para a satisfação profissional e uma possível QV.

\section{Referências}

ALVES, E. F. Qualidade de vida: considerações sobre os indicadores e instrumentos de medida. Revista Brasileira de Qualidade de Vida, Ponta Grossa, v. 03, n. 01, p. 16-23, jan./jun. 2011.

ARAÚJO, G. A.; SOARES, M. J. G. O.; HENRIQUES, M. E. R. M. Qualidade de Vida: percepção de enfermeiros numa abordagem qualitativa. Revista Eletrônica de Enfermagem [internet], v. 11, n. 3, p. 635-641, 2009.

BARDIN, L. Análise de Conteúdo. Lisboa: Edições 70, 1979.

BECK, C. L. C.; BUDÓ, M. L. D.; GONZALES, R. M. B. A qualidade de vida na concepção de um grupo de professoras de enfermagem - elementos para reflexão. Revista da Escola de Enfermagem da USP, São Paulo, v. 33, n. 4, p. 348-354, dez. 1999.

CHIAVENATO, I. Recursos Humanos: o capital humano das organizações. 8. ed. São Paulo: Atlas, 2008.

COFEN - Conselho Federal de Enfermagem. Resolução COFEN 314/2007. Revoga a resolução 276/2003. Regula a concessão a inscrição provisória ao auxiliar de enfermagem. Rio de Janeiro, 30 abr. 2007. Disponível em: <http://site.portalcofen.gov.br/node/4349>. Acesso em: 05 jan. 2012.

COSTA, E. S.; MORITA, I.; MARTINEZ, M. A. R. Percepção dos efeitos do trabalho em turnos sobre a saúde e a vida social em funcionários da enfermagem em um hospital universitário do estado de São Paulo. Cadernos de Saúde Pública, Rio de Janeiro, v. 16, n. 2, p. 553-555, 2000.

DAUBERMANN, D. C.; TONETE, V. L. P. Qualidade de vida no trabalho do enfermeiro da atenção básica à saúde. Acta Paulista de Enfermagem, v. 25, n. 2, p. 277-283, 2012. 
FAKIH, F. T. et al. Perfil dos atendentes de enfermagem frente à lei que regulamenta o exercício da profissão de enfermagem: relato de experiência. Acta Paulista de Enfermagem, São Paulo, v. 12, n. 3, p. 64-69, set./dez. 1999.

FARIAS, S. N. P.; ZEITOUNE, R. C. G. A interferência da globalização na qualidade de vida no trabalho: a percepção dos trabalhadores de enfermagem. Escola Anna Nery Revista de Enfermagem, Rio de Janeiro, v. 8, n. 3, p. 386-392, dez. 2004.

FARIAS, S. N. P.; ZEITOUNE, R. C. G. A qualidade de vida no trabalho de enfermagem. Escola Anna Nery Revista de Enfermagem, Rio de Janeiro, v. 11, n. 3, p. 487-493, set. 2007.

FERNANDES, J. S. et al. Qualidade de vida dos enfermeiros das equipes de saúde da família: a relação das variáveis sociodemográficas. Texto \& Contexto Enfermagem, Florianópolis, v. 19, n. 3, p. 434-442, jul./set. 2010.

FERNANDES, J. S. et al. A relação dos aspectos profissionais na qualidade de vida de enfermeiros das equipes saúde da família. Revista da Escola de Enfermagem da USP, v. 46, n. 2, p. 404-412, 2012.

FOGAÇA, M. C.; CARVALHO, W. B.; MARTINS, L. A. N. Estudo preliminar sobre a qualidade de vida de médicos e enfermeiros intensivistas pediátricos e neonatais. Revista da Escola de Enfermagem da USP, São Paulo, v. 44, n. 3, p. 708-712, 2010.

GORDIA, A. P. et al. Qualidade de vida: contexto histórico, definição, avaliação e fatores associados. Revista Brasileira de Qualidade de Vida, Ponta Grossa, v. 03, n. 01, p. 40-52, jan./jun. 2011.

KIMURA, M.; CARANDINA, D. M. Desenvolvimento e validação de uma versão reduzida do instrumento para avaliação da qualidade de vida no trabalho de enfermeiros em hospitais. Revista da Escola de Enfermagem da USP, São Paulo, v. 43, n. esp., p. 1044-1054, 2009.

LENTZ, R. A. et al. O profissional de enfermagem e a qualidade de vida: uma abordagem fundamentada nas dimensões propostas por Flanagan. Revista Latino-Americana de Enfermagem, Ribeirão Preto, v. 8, n. 4, p. 7-14, ago. 2000.

MASCARENHAS, A.; FERNANDES, R. C. P. Atividades físicas de lazer e distúrbios muscoesqueléticos: revisão de literatura. Revista Baiana de Saúde Pública, Salvador, v. 35, n. 1, p. 9-25, jan./mar. 2011.

MINAYO, M. C. S. O desafio do conhecimento: pesquisa qualitativa em saúde. 12. ed. São Paulo: Hucitec, 2010.

PASCHOA, S.; ZANEI, S. S. V.; WHITAKER, I. Y. Qualidade de vida dos trabalhadores de enfermagem de unidade de terapia intensiva. Acta Paulista de Enfermagem, São Paulo, v. 20, n. 3, p. 305-310, 2007.

REZENDE, M. P. Agravos à saúde de auxiliares de enfermagem resultantes da exposição ocupacional aos riscos físicos. 2003. 114f. Dissertação (Mestrado em Enfermagem) - Programa de Pós-Graduação em Enfermagem, Escola de Enfermagem de Ribeirão Preto - Universidade de São Paulo, Ribeirão Preto. 
ROCHA, S. S. L.; FELLI, U. E. A. Qualidade de vida no trabalho docente de enfermagem. Revista Latino-Americana de Enfermagem, Ribeirão Preto, v. 12, n. 1, p. 28-35, jan./fev. 2004.

SCHMIDT, D. R. C.; DANTAS, R. A. S. Qualidade de vida entre profissionais de enfermagem do bloco cirúrgico. Revista Gaúcha de Enfermagem, Porto Alegre, v. 27, n. 1, p. 100-108, mar. 2006a.

SCHMIDT, D. R. C.; DANTAS, R. A. S. Qualidade de vida no trabalho de profissionais de enfermagem atuantes em unidades de bloco cirúrgico, sob a ótica da satisfação. Revista LatinoAmericana de Enfermagem, Ribeirão Preto, v. 14, n. 1, p. 54-60, jan./fev. 2006b.

TALHAFERRO, B.; BARBOZA, D. B.; DOMINGOS, N. A. M. Qualidade de vida da equipe de enfermagem da central de materiais de esterilização. Revista de Ciências Médicas (Campinas), v. 15, n. 6, p. 495-506, nov./dez. 2006.

THE WHOQOL GROUP. The World Health Organization Quality of Life Assesment (WHOQOL): position paper the world health organization. Social Science and Medicine, v. 41, n. 10, p. 1403-1409. 